\title{
Bound vortex states and exotic lattices in multicomponent Bose-Einstein condensates: The role of vortex-vortex interaction
}

\author{
Davi S. Dantas,,${ }^{1, *}$ Aristeu R. P. Lima, ${ }^{2, \dagger}$ A. Chaves, ${ }^{1, \dagger}$ C. A. S. Almeida, ${ }^{1}$ G. A. Farias, ${ }^{1}$ and M. V. Miloševic ${ }^{3}, \S$ \\ ${ }^{1}$ Departamento de Física, Universidade Federal do Ceará, Caixa Postal 6030, 60455-760 Fortaleza, Ceará, Brazil \\ ${ }^{2}$ Instituto de Ciências Exatas e da Natureza, Universidade da Integração Internacional da Lusofonia Afro-Brasileira, \\ Campus dos Palmares, 62785-000 Acarape, Ceará, Brazil \\ ${ }^{3}$ Departement Fysica, Universiteit Antwerpen, Groenenborgerlaan 171, B-2020, Belgium
}

(Received 10 November 2014; published 27 February 2015)

\begin{abstract}
We numerically study the vortex-vortex interaction in multicomponent homogeneous Bose-Einstein condensates within the realm of the Gross-Pitaevskii theory. We provide strong evidence that pairwise vortex interaction captures the underlying mechanisms which determine the geometric configuration of the vortices, such as different lattices in many-vortex states, as well as the bound vortex states with two (dimer) or three (trimer) vortices. Specifically, we discuss and apply our theoretical approach to investigate intra- and intercomponent vortex-vortex interactions in two- and three-component Bose-Einstein condensates, thereby shedding light on the formation of the exotic vortex configurations. These results correlate with current experimental efforts in multicomponent Bose-Einstein condensates and the understanding of the role of vortex interactions in multiband superconductors.
\end{abstract}

DOI: 10.1103/PhysRevA.91.023630

PACS number(s): 67.85.Fg, 03.75.Mn, 03.75.Lm

\section{INTRODUCTION}

The realization of Bose-Einstein condensates (BECs) has brought about a suitable research environment for investigating the general properties of superfluidity and superconductivity with a high degree of control and versatility [1-3]. This has made vortex states and their dynamics, key concepts for both superfluidity and superconductivity, some of the most investigated topics in low-temperature physics [4-6]. Remarkably, since the first observation of vortices $[7,8]$ and, subsequently, the formation of highly ordered vortex lattices in BECs [9], much theoretical and experimental effort has been made to push the understanding of these systems further. Particularly, recent advances in experimental techniques have led to the realization of condensates with several different types of particle-particle interaction, making this research field even broader. Notably, theoretical results on dipolar [10,11] and spin-orbit-coupled [12] BECs exhibit rich ground-state phases, with bubbles, density stripes, and various vortex lattice geometries. Besides the already mentioned possibilities, other remarkable physical phenomena to be addressed with ultracold atomic systems include, for example, quantum-fluid turbulence in BECs [13], multicharged vortices [14], and vortex-antivortex lattices in superfluid fermionic gases [15].

With the recent prospects of producing condensates with a large number of components and different types of interaction [16,17], vortex states in multicomponent condensates have become even more important. Indeed, recent theoretical results on vortex lattice conformations have shown that multicomponent Bose-Einstein condensates are far from being just a trivial extension of the single-component case. In fact, adding a component brings a multitude of possible configurations

\footnotetext{
*davidantas@fisica.ufc.br

†aristeu@unilab.edu.br

${ }^{\ddagger}$ andrey@fisica.ufc.br

§milorad.milosevic@uantwerpen.be
}

never found in a one-component system, such as amorphous conformations, square lattices, and bound states, including overlapping vortices, vortex dimers, and molecules [18-22]. Some of these conformations suggest that the interaction between vortices can be nonmonotonic with respect to the intervortex distance. Unfortunately, to date, the interaction between vortices in the simplest multicomponent BEC is known only in specific limits of scale by either assuming intervortex distances much greater than the healing length [23] or considering the interaction energy near the vortex peak in the Thomas-Fermi regime [24]. As it turns out, the asymptotic behavior does not account for all conformations found [19-22]. Furthermore, the generalization of these analytical approaches to more complex cases with more components or even with a different kind of intercomponent particle-particle interaction seems to be highly nontrivial.

Within this context, we investigate in the present paper the origin of these unusual quantized vortex states by focusing on the pairwise vortex-vortex interaction $[25,26]$. We consider homogeneous BECs, which possess translation invariance. On the one hand, this is important in itself since the key properties of the experimentally more relevant harmonically trapped BECs in the large particle number regime bear a close resemblance to those of their homogeneous counterparts [27]. On the other hand, the recent achievement of a condensate in a uniform potential [28] enables the experimental verification of our predictions. Experimental progress has already led to the detection of extended phase coherence in a uniform quasi-twodimensional Bose gas [29]. In our approach, constraints are imposed on the Gross-Pitaevskii (GP) formalism, allowing us to fix the vortices in the desired positions for further analysis. In other words, vortices no longer arise naturally from the GP equation itself but are instead placed manually in the position of interest. This brings about the possibility of calculating the interaction energy between vortices as a function of their distance. Then, by investigating conformations which minimize the corresponding energy, we are able to present a 
simple physical picture of the underlying phenomena which lead to vortex states observed elsewhere.

This paper is organized as follows. In Sec. II, we briefly present the main aspects of the theoretical approach, focusing on the Euler-Lagrange equations. Section III summarizes our numerical results and contains a discussion concerning experimental realization in each considered case. Section III A focuses on two-component BECs with contact interaction, and Sec. III B concerns bound states of vortices in twoand three-component condensates also featuring a coherent coupling of the Rabi type. Our concluding remarks are presented in Sec. IV.

\section{THEORETICAL APPROACH}

We begin our consideration with the Gross-Pitaevskii energy functional for an $N_{c}$-component homogeneous BEC. When set into rotation above a given critical frequency, superfluids acquire angular momentum in the form of a vortex [30,31]. In turn, these can be represented by nodes in the wave function $\Psi$ [32]. Here, we adopt a different approach and include vortices directly in the form of a node, disregarding rotation. Therefore, only contributions from the kinetic and interaction energies should enter in the energy functional. Moreover, since vortices will be placed manually in fixed positions, we also do not need to account for the energy provided by the angular momentum of the system, $L_{z}$, which is only necessary to allow vortex formation in the Gross-Pitaevskii theory for a rotating BEC. Then, in the present case, the energy density functional reads

$$
\mathcal{E}=\sum_{\alpha=1}^{N_{c}}\left\{\frac{\hbar^{2}}{2 m_{\alpha}}\left|\nabla \Psi_{\alpha}\right|^{2}+\frac{g_{\alpha}}{2}\left|\Psi_{\alpha}\right|^{4}\right\}+V_{i j},
$$

where the components are labeled by the index $\alpha$. Here, the first and second terms, respectively, account for the usual kinetic and contact interaction energies within each component. In addition, the term $V_{i j}$ stands for the intercomponent coupling energy density.

In what follows, we will be interested in cases where $V_{i j}$ can be written as

$$
V_{i j}=\sum_{i}^{N_{c}} \sum_{j>i}^{N_{c}} g_{i j}\left|\Psi_{i}\right|^{2}\left|\Psi_{j}\right|^{2}-w_{i j}\left(\Psi_{i} \Psi_{j}^{*}+\Psi_{j} \Psi_{i}^{*}\right),
$$

where the first term stands for the contact interaction and the last one represents the Rabi term. The latter characterizes the internal coherent coupling between the components and has already been experimentally implemented by inducing an external driving field, which allows particles to move from one hyperfine spin state to another [33]. These interaction terms are controlled by the parameters $g_{\alpha}, g_{i j}$, and $w_{i j}$. However, it is convenient to redefine them in order to have dimensionless units of energy and length. Therefore, for a two-dimensional system, we introduce the units $\mathcal{E}_{1}=\hbar^{2} \bar{\rho}_{1}^{2} / 2 m_{1}$ for energy density and $\xi=1 / \sqrt{\bar{\rho}_{1}}$ for distances. Here, $\bar{\rho}_{1}$ is the average particle density of the first species. With $E=\mathcal{E} / \mathcal{E}_{1}$ and $\vec{r}=\vec{r}^{\prime} / \xi$, the energy density can be written in its dimensionless form as

$$
\begin{aligned}
E= & \sum_{\alpha=1}^{N_{c}}\left[M_{1 \alpha}\left|\nabla \psi_{\alpha}\right|^{2}+\frac{\gamma_{\alpha}}{2}\left|\psi_{\alpha}\right|^{4}\right] \\
& +\sum_{i}^{N_{c}} \sum_{j>i}^{N_{c}}\left[\gamma_{i j}\left|\psi_{i}\right|^{2}\left|\psi_{j}\right|^{2}-\omega_{i j}\left(\psi_{i} \psi_{j}^{*}+\psi_{j} \psi_{i}^{*}\right)\right],
\end{aligned}
$$

where $\psi_{\alpha}=\xi \Psi_{\alpha}$ is the dimensionless order parameter. This procedure leads to the definition of the mass ratio $M_{1 \alpha}=$ $m_{1} / m_{\alpha}$, the dimensionless contact interaction strengths $\gamma_{\alpha}=$ $g_{\alpha} \bar{\rho}_{1}^{2} / \mathcal{E}_{1}$ and $\gamma_{i j}=g_{i j} \bar{\rho}_{1}^{2} / \mathcal{E}_{1}$, and the dimensionless Rabi frequencies $\omega_{i j}=w_{i j} \bar{\rho}_{1} / \mathcal{E}_{1}$. The contact parameters can be well controlled in experiments by means of Feshbach resonances, and appropriate values should enable the visualization of the properties of interest.

In order to measure the interaction potential between two vortices, we consider the total energy as a function of the intervortex distance. This is justified because the system is free from external contributions, so that the vortex position affects only the vortex-vortex interaction. Moreover, calculating the system energy as a function of the distance between vortex pairs requires fixing the vortex, which is achieved by fixing the $2 \pi$ whirl of the phase of the condensate wave function, thereby introducing a node in it. For one vortex in the $\alpha$ component, for example, the wave function is given as $\psi_{\alpha}(x, y)=\sqrt{N_{\alpha}} e^{i n_{k} \theta_{k}} f_{\alpha}(x, y)$, with $N_{\alpha}$ being the number of particles in the $\alpha$ component and $n_{k}$ being the corresponding winding number, i.e., the quanta of circulation carried by the vortex. In addition, the angle $\theta_{k}$ is defined around the locus of the $k$ th vortex. Correspondingly, for two vortices, we have $\psi_{\alpha}(x, y)=\sqrt{N_{\alpha}} e^{i n_{1, \alpha} \theta_{1, \alpha}} e^{i n_{2, \alpha} \theta_{2, \alpha}} f_{\alpha}(x, y)$. Since such a vortex dimer structure is not circularly symmetric, Cartesian coordinates are an appropriate choice, with $e^{i n_{k, \alpha} \theta_{k, \alpha}}$ written as

$$
e^{i n_{k, \alpha} \theta_{k, \alpha}}=\left(\frac{x_{k, \alpha}+i y_{k, \alpha}}{x_{k, \alpha}-i y_{k, \alpha}}\right)^{n_{k, \alpha} / 2},
$$

where $\vec{r}_{k, \alpha}=\left(x_{k, \alpha}, y_{k, \alpha}, 0\right)$ stands for the in-plane position vector with the origin at the center of the vortex $k$. As a consequence of the fixed circular phase around the vortex, singularities in the amplitudes appear naturally from the energy functional minimization.

We remark that the present ansatz is general enough to allow for considering components whose vortices might differ both in position and in winding number. This is important in order to obtain the total energy of the system in the presence of the intercomponent coupling as a function of the relative distance of the vortices and then verify whether one has found the lowest-energy configuration or not. Indeed, in the absence of intercomponent coupling $\gamma_{i j}=\omega_{i j}=0$, we have found that the single-vortex state is infinitely degenerate and the respective position of vortices in different components is irrelevant. This supports our statement above that the total energy depends only on the intervortex distance. In the presence of an intercomponent coupling, however, vortices in different components will rather stay on top of each other (separate) for attractive (repulsive) effective vortex-vortex interaction potentials. 
With an adequate ansatz for the condensate wave function at hand, we minimize the total energy, which is obtained by integrating in space the quantity $E-\sum \mu_{\alpha}\left|\psi_{\alpha}\right|^{2}$ with respect to $f_{\alpha}$. Here, $\mu_{\alpha}$ stands for the chemical potential of the $\alpha$ component and is introduced to keep the corresponding number of particles constant, which means that we are not taking population transfer between different hyperfine spin states into account. The minimization process is implemented by numerically solving the set of $N_{c}$ Euler-Lagrange equations

$$
\begin{aligned}
& M_{1 \alpha} K_{\alpha} f_{\alpha}+\mu_{\alpha} f_{\alpha}-\gamma_{\alpha} N f_{\alpha}^{2} f_{\alpha} \\
& +\sum_{i}^{N_{c}} \sum_{j>i}^{N_{c}} \omega_{i j} \Theta_{i j}\left(f_{i} \delta_{\alpha, j}+f_{j} \delta_{\alpha, i}\right) \\
& -N \gamma_{i j}\left(f_{j}^{2} f_{i} \delta_{i, \alpha}+f_{i}^{2} f_{j} \delta_{j, \alpha}\right)=0,
\end{aligned}
$$

where

$$
\Theta_{i j}=\cos \left[\left(n_{1 i} \theta_{1 i}-n_{1 j} \theta_{1 j}\right)+\left(n_{2 i} \theta_{2 i}-n_{2 j} \theta_{2 j}\right)\right] .
$$

At this point, we have chosen the same number of particles for all components by setting $N_{\alpha}=N$. Notice that Eq. (5) has the same form of the Gross-Pitaevskii equation, and we shall call it the constrained GP equation (CGP). One important difference, however, should be emphasized: the term

$$
K_{\alpha}=\nabla^{2}-\left({\overline{X_{\alpha}}}^{2}+{\overline{Y_{\alpha}}}^{2}\right),
$$

which arises from the kinetic contribution of the energy density functional, carries two extra terms, $\overline{X_{\alpha}}$ and $\overline{Y_{\alpha}}$, allowing us to manually set the coordinates of fixed vortices as well as their winding numbers. These terms are given by

$$
\overline{X_{\alpha}}=\frac{n_{1, \alpha} x_{1, \alpha}}{r_{1, \alpha}^{2}}+\frac{n_{2, \alpha} x_{2, \alpha}}{r_{2, \alpha}^{2}}, \quad \overline{Y_{\alpha}}=\frac{n_{1, \alpha} y_{1, \alpha}}{r_{1, \alpha}^{2}}+\frac{n_{2, \alpha} y_{2, \alpha}}{r_{2, \alpha}^{2}} .
$$

Finally, we remark that a similar procedure has already been successfully applied to superconductors in the framework of the Ginzburg-Landau theory $[25,26]$.

\section{NUMERICAL RESULTS}

The numerical solution of the CGP equations is obtained using a two-dimensional rectangular system divided into a uniform square grid $4000 \times 2000$ with the total dimension $1000 \xi \times 500 \xi$ using the finite-difference technique and a relaxation method suitable for nonlinear differential equations. This leads us to the lowest-energy vortex structure that satisfies the constraint that vortices are placed in fixed positions. The obtained condensate amplitudes are then substituted back into the energy density, which is numerically integrated, yielding the total energy $U=\int E\left(\psi_{\alpha}\right) d x d y$ (dimensionless in units of $\left.E_{1}=\mathcal{E}_{1} \xi^{2}\right)$ of the corresponding vortex configuration. With the total energy as a function of the vortex-vortex distance at hand, our theoretical approach will then be used to investigate vortex-state solutions of the Gross-Pitaevskii equation in the presence of rotation, reflecting the real experimental conditions under which vortices are generated.

In what follows, we present the results first for BECs with only contact interaction, where we focus on the twocomponent case, and then also for coherently coupled twoand three-component BECs. For simplicity, we took the same number of particles per component for all situations,

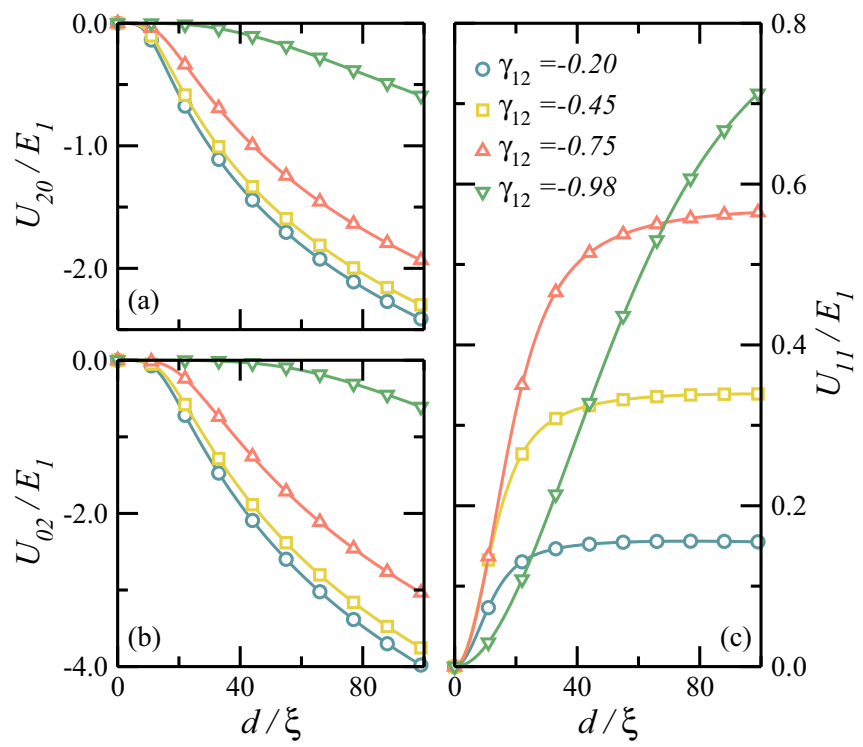

FIG. 1. (Color online) Vortex-vortex interaction potentials, considering $M_{12}=2.0$, for (a) and (b) two vortices in the same component and (c) vortices in different components. Symbols denote different intercomponent coupling strengths: $\gamma_{12}=-0.20$ (blue circles), $\gamma_{12}=-0.45$ (yellow squares), $\gamma_{12}=-0.75$ (red triangles), and $\gamma_{12}=-0.98$ (green downward triangles).

$N=5 \times 10^{4}$, and set the intracomponent couplings equal to each other $\left(\gamma_{1}=\gamma_{2}=\gamma_{3}=1\right)$. Since the investigation of the vortex-vortex interaction is performed within the miscibility condition $\left(\gamma_{1} \gamma_{2}-\gamma_{12}^{2}>0\right)$, the intercomponent coupling parameters used are within the upper and lower limits $\left|\gamma_{12}\right|<1$. We denote by $U_{i, j, k}$ the total energy for the case of $i$ vortices placed in the first component, $j$ vortices placed in the second, and $k$ vortices placed in the third (if considered).

\section{A. Two-component BEC with contact interaction}

Let us first consider the case of a two-component condensate, with two vortices placed at $(x, y)=( \pm d / 2,0)$ so that the distance between them is $d$. We shall consider components with different masses, as this has been found to lead to unusual lattice conformations in Ref. [19]. By assuming a mass ratio of $M_{12}=2.0$ between the different components, we plot in Fig. 1 the vortex-vortex interaction for different values of the intercomponent coupling strength: $\gamma_{12}=-0.20$ (circles), $\gamma_{12}=-0.45$ (squares), $\gamma_{12}=-0.75$ (triangles), and $\gamma_{12}=-0.98$ (downward triangles). Figures 1(a) and 1(b) represent the intracomponent vortex-vortex interaction for the first and second components, respectively, whereas Fig. 1(c) depicts the intercomponent vortex-vortex interaction.

It turns out that for a two-vortex configuration, the interaction potential is always a monotonic function of the distance, where the attractive or the repulsive behavior is determined by the properties of the particle-particle interaction. Nonetheless, one interesting feature can already be identified at this level: for intercomponent attraction, increasing the corresponding interaction strength $\left|\gamma_{12}\right|$ weakens the intracomponent vortexvortex repulsion. This can be understood in terms of the form of the amplitudes $f(x, y)$. Indeed, as the attractive intercomponent 

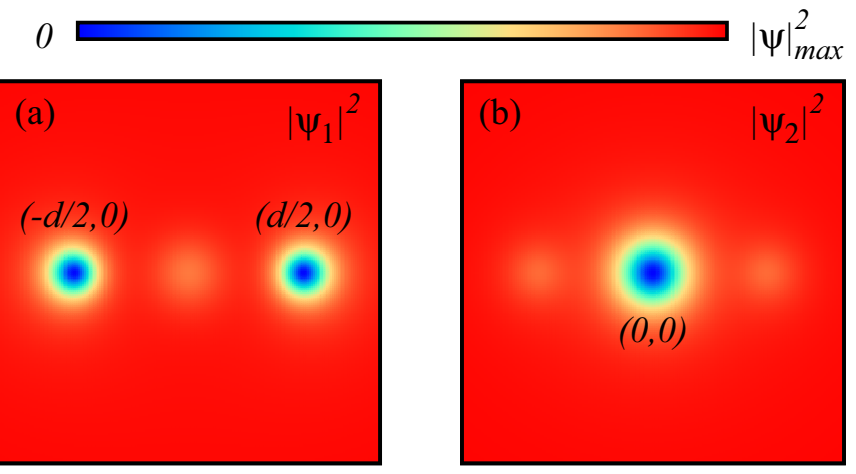

FIG. 2. (Color online) The occupation number density contour plots for (a) the first component with two vortices positioned at $(-d / 2,0)$ and $(d / 2,0)$ and (b) the second component with a single vortex in the center of the mesh. Notice the depleted density in one component in the positions where the other component features a vortex. The investigation of the system energy with respect to $d$ for this conformation enables us to identify possible bound states in a two-component Bose-Einstein condensate with mass ratio $M_{12}=2.0$.

interaction becomes stronger, the energy is lowest for the largest possible superposition of the amplitudes, leading to a decrease in the density of one component in the spatial region where the other one has a vortex. Consequently, as $\left|\gamma_{12}\right|$ increases, these depletions should become more akin to vortices, and the repulsive nature of the intracomponent vortex-vortex interaction is softened by the depletion-vortex attractive interaction.

Before we explore the consequences of the depletion induced in one component due to the presence of vortices in the other one, let us turn our attention to the mass ratio $M_{12}$. According to the Feynman relation of the vortex density in a rotating superfluid [32], the vortex density is proportional to the particle mass, according to

$$
n_{v, \alpha}=\frac{m_{\alpha} \Omega_{R}}{\pi \hbar},
$$

where $\Omega_{R}$ is the angular rotation frequency of the condensate. Thus, for a mass ratio of $M_{12}=2.0$, it is natural to investigate situations where two vortices (one vortex) are placed in the heavier (lighter) component. To this end, we have pinned a vortex in the second component in the center of the mesh and have placed two additional vortices in the first component, separated by a distance $d$, as illustrated in Fig. 2 by a contour plot of both components. In Fig. 2, colors (shading) represent the number density for both order parameters, and vortex coordinates are shown as a function of the intervortex distance $d$. In this case, the system energy is no longer a vortex-vortex interaction potential. However, this conformation should enable the identification of possible bound states provided by the competition between the intercomponent and intracomponent interactions, such as dimers and giant vortices [34-41] in the same component. Notice the weaker density in one component in the positions where the other component features a vortex, characterizing the depletion effect discussed before.

The total energy of this three-vortex configuration is plotted in Figs. 3(a) and 3(b), whereas Figs. 3(c)-3(f) demonstrate the

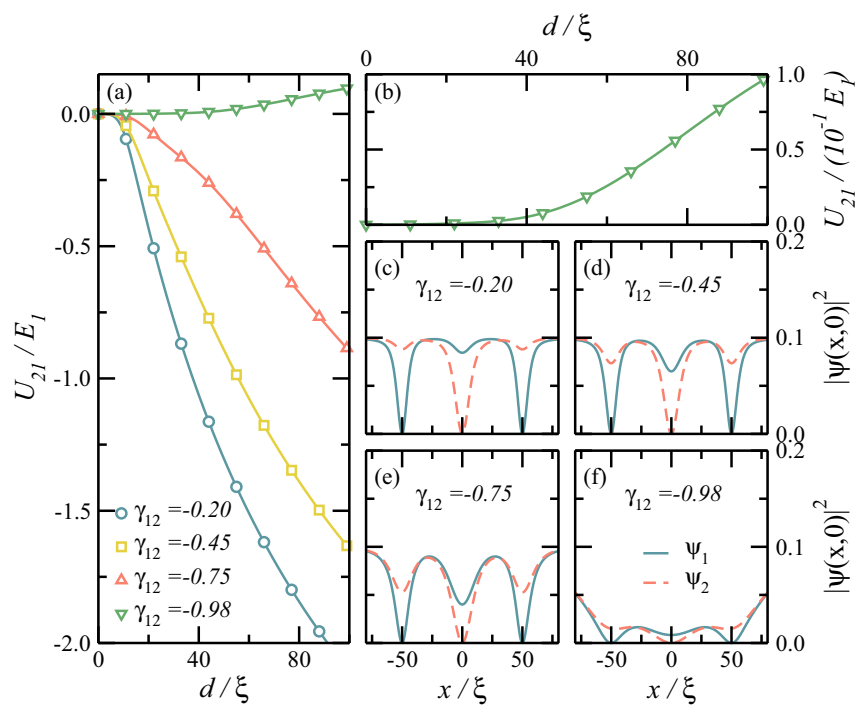

FIG. 3. (Color online) (a) Total energy for the three-vortex configuration $U_{21}$, characterizing a binary system with $M_{12}=2$. The vortex in the second component is fixed in the center of the mesh, whereas vortices in the first component are placed at symmetric positions separated by distance $d$. (b) A magnification of the case with $\gamma_{12}=-0.98$ shows that in the favored configuration, the two vortices in the first component are located on top of each other and on top of the vortex in the second component. (c)-(f) Occupation number density of both components, considering vortices separated by $100 \xi$ for all cases considered in (a), where the solid (dashed) line stands for the first (second) component.

previously discussed density depletion for several values of the relative interaction strength.

From Fig. 3(a), one sees that for low values of $\left|\gamma_{12}\right|$ the energy is a monotonically decreasing function with the distance $d$, indicating repulsion between the vortices in the same component. However, this behavior is completely changed for $\gamma_{12}=-0.98$, where the total energy turns into a monotonically increasing function, allowing vortices of the same component to occupy the same position, thus forming a giant vortex. Indeed, as one can see in the magnification of this result in Fig. 3(b), the energy minimum corresponds to a vanishing distance between the vortices in the same component. At this point, it is evident that the depletion effect is the main cause of the behavioral change of the effective interaction. In fact, the strong coupling between the components makes the intracomponent repulsive contribution less relevant than the intercomponent vortex-vortex interaction, and the attractive effective interaction is achieved for every distance $d$. In Figs. 3(c)-3(f), the occupation number density for vortices separated by $100 \xi$ shows the depletion effect, where depletions become more akin to vortices for higher values of intercomponent particle coupling $\left|\gamma_{12}\right|$. It is noticed that vortices become larger when $\gamma_{12}$ is increased, causing the strong vortex overlap illustrated in Fig. 3(f). This effect depends on the intercomponent particle coupling and vortex density.

Before going into further details concerning the vortex core, let us first analyze how the effective interaction between vortices changes from repulsive to attractive by considering 

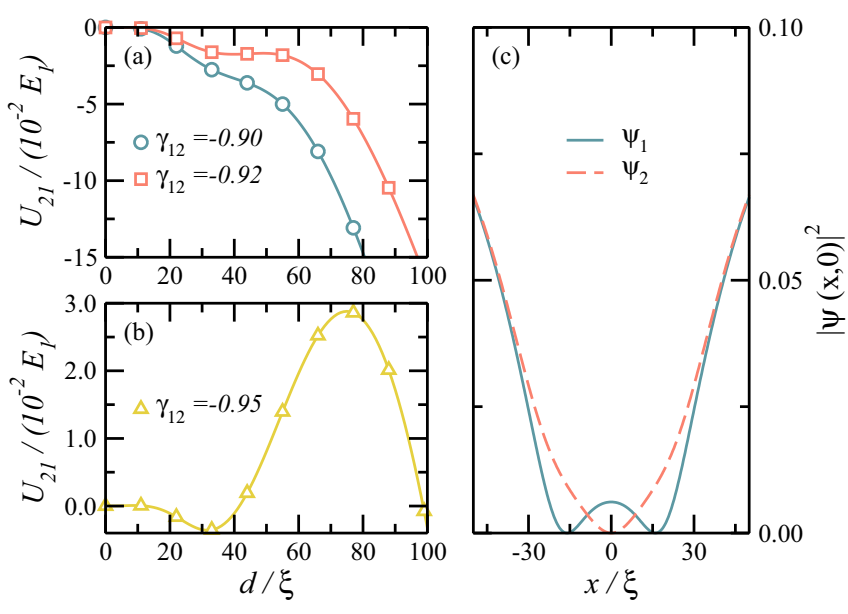

FIG. 4. (Color online) (a) Total energy of a three-vortex structure in a two-component BEC for $\gamma_{12}=-0.90$ (blue circles) and -0.92 (red squares). The curve bumps may lead to nontriangular lattices, despite the overall repulsive behavior. (b) A triple-vortex structure with $\gamma_{12}=-0.95$. The minimum far from the origin allows for the formation of dimers for specific vortex densities. (c) Dimer configuration profile for $\gamma_{12}=-0.95$.

intermediate values of $\gamma_{12}$ between $\gamma_{12}=-0.75$ and $\gamma_{12}=$ -0.98 . We have found that, for $\gamma_{12}=-0.90$ and $\gamma_{12}=-0.92$, as illustrated in Fig. 4(a), the energy curves still have repulsive characteristics, but they exhibit shoulders at finite distances, which could indicate the formation of nontriangular lattices in these minima of the interaction potential, despite the overall repulsive behavior. In Fig. 4(b), by setting $\gamma_{12}=-0.95$, we have obtained a total energy which exhibits a nonmonotonic behavior and acquires a clear minimum at finite intervortex distance. This strongly indicates that the presence of a vortex in one component can cause the formation of a bound state of two vortices (dimer) in the heavier component, as illustrated in Fig. 4(c). These results corroborate the states obtained in the numerical experiments of Ref. [19], in which the Abrikosov lattice of vortex dimers in one component, sitting on single vortices in the other, was found in a binary mixture.

In addition to effects caused in the vortex-vortex interactions, which lead to different lattice conformations, the depletion effect also leads to the formation of vortices with different core sizes depending on the choice of the $\gamma_{12}$ parameter. Actually, this was pointed out before in Figs. 3(c)-3(f), where more negative values of $\gamma_{12}$ result in vortices with larger core sizes. To investigate this dependence, by setting a vortex in only one of the two components, the vortex core radius is measured in Fig. 5 for different values of intercomponent coupling, between $\gamma_{12}=-0.90$ and $\gamma_{12}=0.90$. This was done by measuring the distance between the center of the vortex and the point at which the order parameter decreases by half of its long-range convergence value.

Since the components have different masses, in Fig. 5 we investigated this effect for vortices in both components, where the core size for a first- (second-) component vortex is presented by the solid (dashed) line. It is observed that the more the two components are coupled, the larger the vortex radii will be. This effect actually comes from the depletion originated by the vortex depletion which contributes to an increased vortex

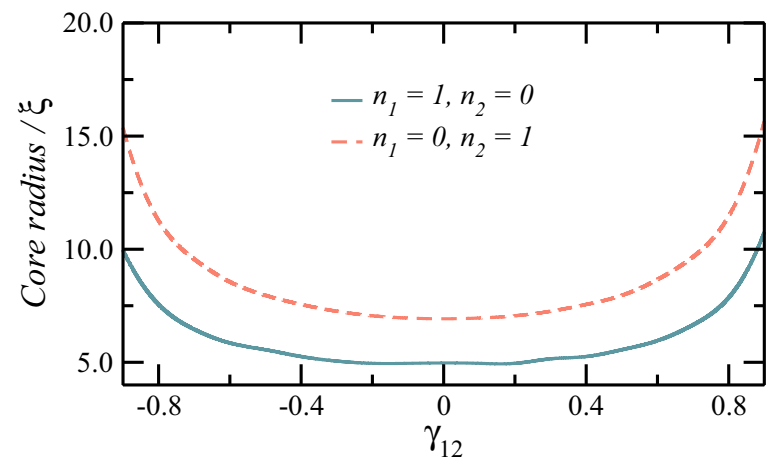

FIG. 5. (Color online) Vortex core size as a function of the intercomponent coupling for $M_{12}=2.0$. The blue solid line (red dashed line) represents the core radius of a vortex in the first (second) component.

size. For both components, this behavior is qualitatively the same, suggesting that the different masses cause simply a shift between two curves.

Let us at this point address the question of how realistic the present predictions are from an experimental point of view. For example, a two-species BEC with a tunable interspecies interaction has already been produced from the mixture of ${ }^{87} \mathrm{Rb}$ and ${ }^{41} \mathrm{~K}$, for which one has $M_{12} \approx 2.1$ [42]. In that particular study, a Feshbach resonance around a magnetic field of $B \approx$ $79 \mathrm{G}$ allows for tuning $a_{12}$ from positive to negative values. For example, for $80.7 \mathrm{G}, a_{12} \approx-185 a_{0}$ is reported, with $a_{0}$ being the Bohr radius. This clearly opens up the possibility of obtaining the appropriate negative values of $\gamma_{12}$ needed to experimentally observe the predicted vortex states. We also remark that, for a ${ }^{87} \mathrm{Rb}_{-}{ }^{85} \mathrm{Rb}$ mixture, the tunability of the interspecies interaction has already been used to probe various mean-field regimes such as spatial separation as well as the formation of long-lived droplets (see Ref. [43]).

\section{B. Vortex dimers and trimers in coherently coupled BECs}

In this section, we consider the presence of an internal coherent coupling of the Rabi type between the different components. In order to do so, an extra term should be added to the energy density functional, as pointed out in Sec. II. For a system with $N_{c}$ components, the Rabi contribution in Eq. (2) can be rewritten in the form

$$
-2 \sum_{i}^{N_{c}} \sum_{j>i}^{N_{c}} \omega_{i j}\left|\psi_{i}\right|\left|\psi_{j}\right| \cos \left(\theta_{i}-\theta_{j}\right) .
$$

If the signs of $\omega_{i j}$ coefficients are all positive, the ground-state energy is achieved when all the phases $\theta_{i}$ are the same. Recalling that the phases fix the position of the vortices, this means that vortices of different components would overlap, featuring an attractive potential. On the other hand, by setting positive values for the contact coupling $\gamma_{i j}$, a repulsive intercomponent vortex-vortex interaction emerges. The balance between these two types of interaction can lead to molecular conformations of vortices. Unlike the previous case, these bound states do not arise due to the mass difference between the components but due to the competing interactions induced by the Rabi contribution. To illustrate the above argument, consider, for 


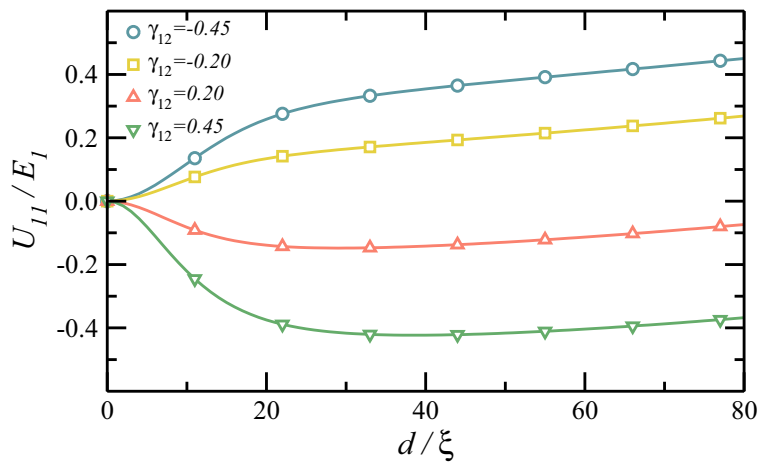

FIG. 6. (Color online) Two-vortex intercomponent interaction potential in the case of competing contact and Rabi coupling, $\omega=2.1 \times 10^{-5}$ and $M_{12}=1$, for several values of $\gamma_{12}$.

instance, the case with two components with equal masses $M_{1 \alpha}=1$, with one vortex in each component.

As illustrated in Fig. 6, a vortex dimer may be formed in the case of a short-range repulsive and long-range attractive intercomponent potential, as observed for positive values of $\gamma_{i j}$, where a minimum of interaction energy is found at finite intervortex distance due to a competition between two types of intercomponent interactions. On the other hand, negative values of $\gamma_{12}$ lead to an extended attractive interaction in the long range.

Let us now address the question of the formation of vortex trimers in a three-component condensate, i.e., bound states consisting of three vortices. In an attempt to reduce the excessive number of parameters, we shall consider the case with $\omega_{12}=\omega_{13}=\omega_{23}=\omega$ and $\gamma_{12}=\gamma_{13}=\gamma_{23}=\gamma$. This choice also helps in finding the minimum-energy configuration since in this case, for symmetry reasons, it is expected that the three vortices are arranged equidistantly. Thus, we manually placed the vortices at the vertices of an equilateral triangle with sides with length $d$, whose loci then read $S_{1}=(0, \sqrt{3} d / 4)$, $S_{2}=(-d / 2,-\sqrt{3} d / 4)$, and $S_{3}=(d / 2,-\sqrt{3} d / 4)$. This is illustrated in Fig. 7, where contour plots of the densities of the three components are shown. Notice the presence of the depletion effect in this case as well. Subsequently, we investigated the minimum-energy conformation as a function of $d$ for several values of $\omega$.

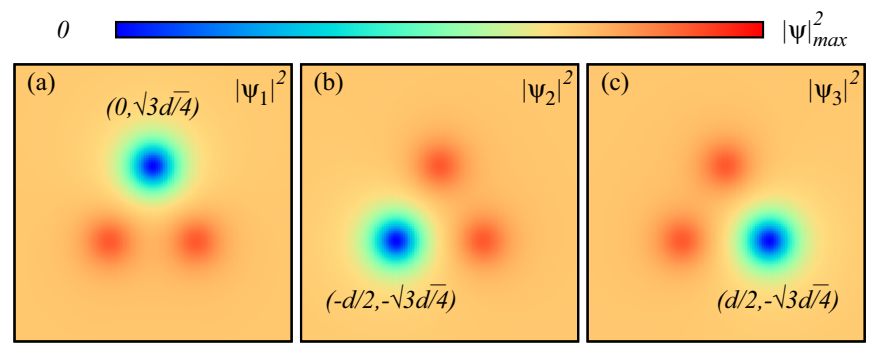

FIG. 7. (Color online) The occupation number density contour plots of (a) the first component with a vortex positioned at $(0, \sqrt{3} d / 4)$, (b) the second component with a vortex positioned at $(-d / 2,-$ $\sqrt{3} d / 4$ ), and (c) the third component with a vortex positioned at $(d / 2,-\sqrt{3} d / 4)$.
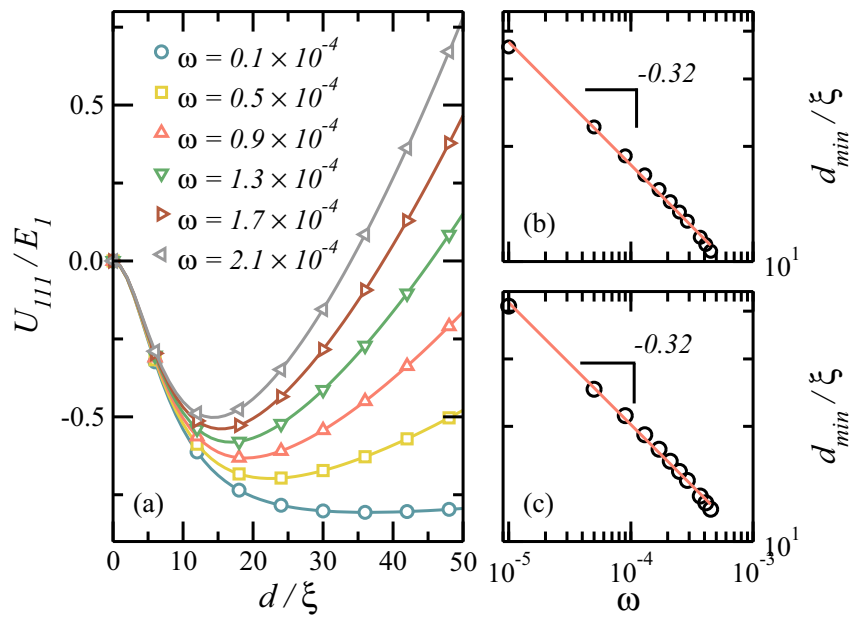

FIG. 8. (Color online) (a) Total energy $U_{111}$ for the equilateraltriangle vortex configuration as a function of the side $d$ for $\gamma=$ 0.45 and for several values of $\omega$. (b) Plot of the optimal side of the equilateral vortex triangle vs $\omega$ for $\gamma=0.45$. The red line represents a power law with an exponent of -0.3214 and a coefficient of 0.9265 . (c) Same as (b) but for $\gamma=0.60$. The red line represents a power law with an exponent of -0.3186 and a coefficient of 1.0746 .

In Fig. 8(a), where we consider $\gamma=0.45$, there is indeed a finite value $d_{\min }$ for the length of the triangle's sides that minimizes the interaction energy $U_{111}$. Moreover, Figs. 8(b) and 8(c) display power-law dependencies of $d_{\min }$ in $\omega$ for $\gamma=$ 0.45 and $\gamma=0.60$, respectively, which explains the vortex trimer configurations observed, e.g., in Fig. 3 of Ref. [20]. Despite the fact that the exponents are quite similar in the two considered cases, they should depend on both $\gamma$ and the number of particles $N$. Indeed, considering the extreme case where $\gamma$ vanishes, the inflection point of the vortex interaction should always be at $d=0$ for any value of $\omega$, leading to a vanishing exponent in the power-law dependence $d_{\min }(\gamma)$.

Since the geometry was imposed from the beginning by setting the vortices at the vertices of the equilateral triangle, the existence of a value $d_{\min }$ which minimizes the total energy does not guarantee that this configuration corresponds to the ground state. In order to allow for different configurations and thereby check if the equilateral triangle is really a minimum of the energy, we have allowed for different positions of the vertex $S_{1}$ while keeping $S_{2}$ and $S_{3}$ fixed for $\omega=1.7 \times 10^{-4}$ and $\gamma=0.45$. As can be seen from Fig. 9, the equilateral-triangle configuration turns out to be stable, corresponding to at least a local minimum of the interaction energy.

Although not very common, there are other ways to obtain bound vortex states which do not arise from a competition between different types of interaction. In fact, it is also possible to obtain dimers and trimers exclusively from Rabi coupling in condensates with at least three components. These vortex states arise from frustration between the phase-locking tendencies. As mentioned previously, a set of positive Rabi frequencies $\omega_{12}=\omega_{13}=\omega_{23}=\omega>0$ would lead to $\theta_{1}=\theta_{2}=\theta_{3}$ as a minimum of the energy, causing the same spatial occupation for all three vortices. However, by assuming, for example, $\omega_{12}=\omega_{13}=-\omega_{23}=\omega$, it is not possible to satisfy the minimization energy condition $\cos \left(\phi_{1}-\phi_{2}\right)=\cos \left(\phi_{1}-\phi_{3}\right)=1$ 

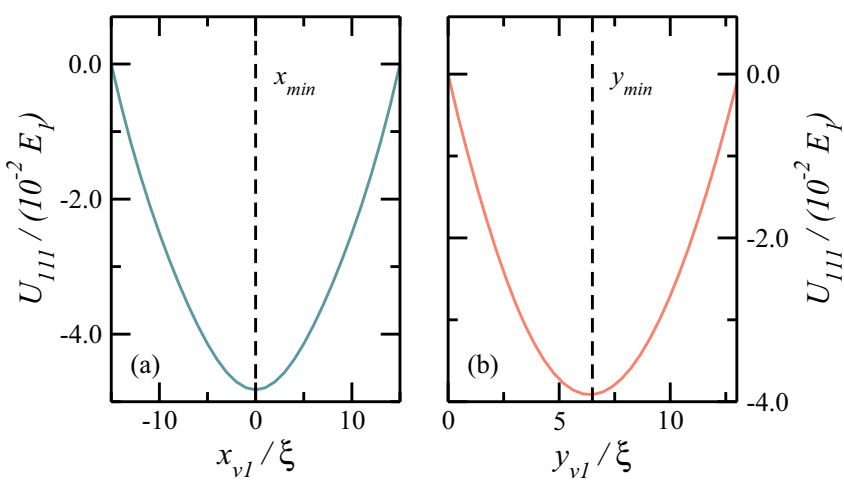

FIG. 9. (Color online) (a) Total energy $U_{111}$ as a function of the displacement of the first component vortex $x_{v 1}$ from the $S_{1}$ position in the $x$ direction. (b) Total energy $U_{111}$ as a function of the displacement of the first component vortex $y_{v 1}$ from the origin in the $y$ direction. For both cases, we have considered $\gamma=0.45$ and $\omega=1.7 \times 10^{-4}$. The vertical dashed line represents the coordinates of the first component vortex for the minimum-energy equilateral-triangle configuration.

and $\cos \left(\phi_{2}-\phi_{3}\right)=-1$ simultaneously, which characterizes a frustrated system [44-47].

Since the frustration phenomenon arises basically from the problem of minimizing the total energy with respect to the order-parameter phases, we must redefine our ansatz, adding extra phases to our order parameters in order to account for the phase tendencies which minimize the energy. Thus, by assuming an ansatz of the form $\psi_{n}(x, y)=\sqrt{N} e^{i \theta_{n}} e^{-i \phi_{n}} f_{n}(x, y)$, the consequences of choosing Rabi frequencies which lead to a frustrated system were investigated for $\omega_{12}=\omega_{13}=-\omega_{23}=$ $\omega$ and $\gamma_{12}=\gamma_{13}=\gamma_{23}=0$, where we have considered $\phi_{1}=$ $\phi_{2}=0$ and several values for $\phi_{3}$.

In Fig. 10(a), we pinned vortices of the first and second components on top of each other in the center of the mesh and displaced the vortex of the third component at a distance $d$ from the origin. This procedure was made for different values of phase $\phi_{3}$. The obtained results show a potential that suggests the formation of a bound vortex state, with the third-component vortex outside the origin, for $\phi_{3}=0$, where the potential minimum is at $d \approx 4 \xi$. This conformation arises from the competition between the attraction with the vortex of the first component and the repulsion with the vortex of the second component. By symmetry, the same result should also appear for $\phi=\pi$. This configuration has the lowest energy among the other investigated cases; however, we cannot guarantee that this conformation is the ground state. Actually, finding the ground-state conformation would require varying all three extra phases and also other vortex positions in the grid, which is very expensive computationally and is left for future investigation. Nevertheless, these results are sufficient to ensure that vortex molecules are stable states. In Fig. 10(b), we show that increasing the Rabi coupling makes the frustration effect even more robust and leads to a deeper minimum in the interaction potential.

In view of the present predictions concerning Rabi-coupled BECs, it becomes important to consider the possibilities of experimental realization of adequate samples. In that respect, we focus on the phenomena of interest, namely, Rabi coupling and homogeneous confinement. They have both already been

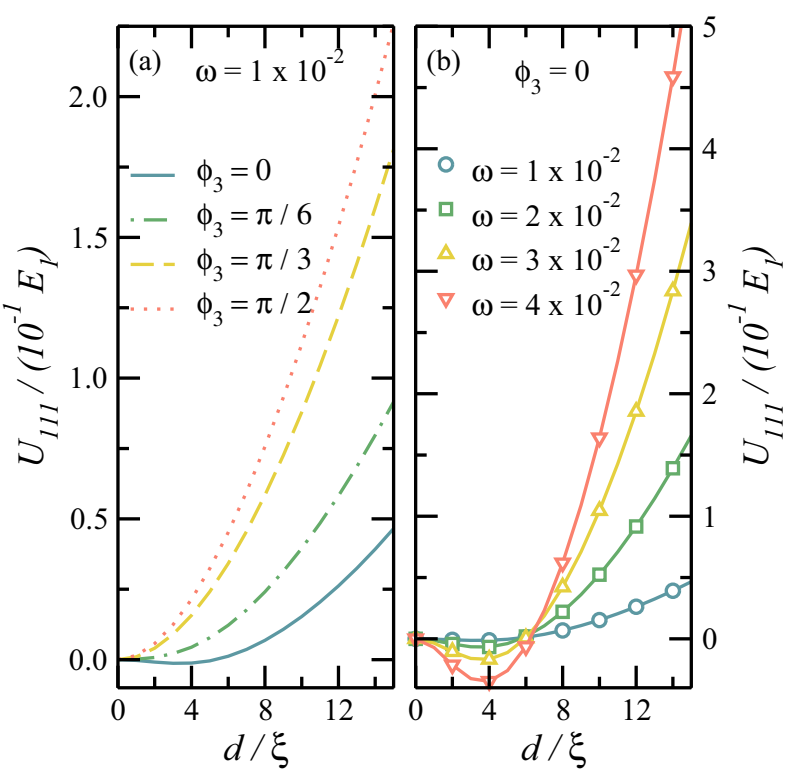

FIG. 10. (Color online) (a) Total energy $U_{111}$, with vortices of the first and second components pinned in the center of the mesh, as a function of the third component vortex distance $d$ from the origin for different values of the third component extra phase $\phi_{3}: 0$ (solid blue line), $\pi / 6$ (green dash-dotted line), $\pi / 3$ (yellow dashed line), and $\pi / 2$ (red dotted line), with $\omega_{12}=\omega_{13}=-\omega_{23}=1 \times 10^{-2}$. (b) Total energy $U_{111}$ for the same conformation as in (a), keeping $\phi_{3}=0$ and assuming different values of $\omega: \omega=1 \times 10^{-2}$ (blue circles), $\omega=2 \times 10^{-2}$ (green squares), $\omega=3 \times 10^{-2}$ (yellow upward triangles), and $\omega=4 \times 10^{-2}$ (red downward triangles).

experimentally achieved with ${ }^{87} \mathrm{Rb}$. Before we turn to the specific experimental setups, we recall that in our calculations the parameter governing the Rabi coupling is $\omega=w \bar{\rho}_{1} / \mathcal{E}_{1}$, where $w=\hbar \Omega$ relates the Rabi energy $w$ to the Rabi oscillation frequency $\Omega$.

We first consider a recent study in Ref. [29] that demonstrates long-range coherence in quasi-two-dimensional Bose gases. There, two-dimensional densities of about a few hundred ${ }^{87} \mathrm{Rb}$ atoms per square micrometer are achieved and combined with harmonic trapping in the third direction whose frequency $v_{z}$ ranges from 350 up to $1500 \mathrm{~Hz}$. Moreover, for ${ }^{87} \mathrm{Rb}$, the (three-dimensional) $s$-wave scattering length is $a_{s} \approx 100 a_{0}$, with $a_{0}$ being the Bohr radius. For the values of the Rabi couplings we turn to Ref. [33], where Rabi oscillation frequencies of the order $2 \pi \times 10^{2} \mathrm{~s}^{-1}$ have been realized. We estimate the Rabi coupling parameter $\omega$ by calculating $E_{1}=\xi^{2} \mathcal{E}_{1}$ for these values of the particle density and Rabi frequencies, arriving at $\omega=\frac{2 m_{1} \Omega}{\hbar \bar{\rho}_{1}}=\frac{2}{a_{\mathrm{R}_{a b i}}^{2} \bar{\rho}_{1}} \approx 1.7 \times 10^{-2}$, where we have associated a length $a_{\mathrm{R} a b i}=\sqrt{\hbar / m_{1} \Omega}$ with the Rabi oscillation. This value is already higher than ones needed for the effects discussed in this paper.

It is also possible to improve the estimates of the actual experimental values of the coherent coupling by including the effect of the transversal harmonic trapping, rendering the calculation more realistic. Indeed, in order to obtain typical values of $\omega$ in quasi-two-dimensional systems, one should correct the $s$-wave scattering parameter $g$ for the freezing of the third dimension. This is done by dividing the three-dimensional 
$g_{3 D}=4 \pi \hbar^{2} a_{s} / m_{1}$ by $\sqrt{2 \pi} a_{z}$, with $a_{z}$ being the oscillator length in the third dimension [48]. If one then associates the energy $E_{1}$ with the contact interaction energy $g \bar{\rho}_{1}$, a slightly different expression for the coherence parameter emerges, $\omega=\frac{1}{a_{\mathrm{R}_{a b i}^{2} \bar{\rho}_{1}}} \frac{a_{z}}{\sqrt{8 \pi} a_{s}} \approx 1 \times 10^{-1}$. Notice that, besides the fact that this value of $\omega$ is even higher in quasi-two-dimensional traps, this expression allows us to identify the trap frequency in the third dimension as well as the Rabi oscillation frequency and the particle density as tuning knobs for studying the effects of coherent coupling in multicomponent BECs.

\section{CONCLUSION}

In summary, we have presented a method for calculating pairwise vortex-vortex interaction potentials in multicomponent Bose-Einstein condensates that is capable of revealing the underlying reasons for the unusual vortex configurations, such as different lattice geometries and few-vortex clusters. We applied our theory to specific examples and thereby clarified the formation of a lattice of dimers in rotating two-component BECs as well as the dimer (trimer) state in a coherently coupled two- (three-) component BEC by pointing out the role of the nonmonotonic interaction potential with respect to the intervortex distance in both cases. Our analysis of the present-day experimental capabilities, combined with simple estimates of relevant parameters, indicates that the considered effects are on the verge of being directly observed in the lab. We remark that the present theory can be straightforwardly adapted to any number of condensate components, providing a tool to anticipate different aspects of vortex physics in Bose-Einstein condensates. As a matter of fact, even for one-component BECs featuring the anisotropic and longrange dipolar interaction, vortex phases such as bubbles and stripes have been foreseen $[10,11,49]$, for which a thorough understanding of the role of vortex-vortex interaction is still lacking.

\section{ACKNOWLEDGMENTS}

This work was supported by the National Council for Scientific and Technological Development (CNPq-Brazil), the Coordination for the Improvement of Higher Education Personnel (CAPES-Brazil), Research Foundation Flanders (FWO), and the bilateral FWO-CNPq program between Flanders and Brazil. M.V.M. acknowledges support from the CAPES-PVE program (Grant No. BEX1392/11-5).
[1] M. H. Anderson, J. R. Ensher, M. R. Matthews, C. E. Wieman, and E. A. Cornell, Science 269, 198 (1995).

[2] C. C. Bradley, C. A. Sackett, J. J. Tollett, and R. G. Hulet, Phys. Rev. Lett. 75, 1687 (1995).

[3] K. B. Davis, M. O. Mewes, M. R. Andrews, N. J. van Druten, D. S. Durfee, D. M. Kurn, and W. Ketterle, Phys. Rev. Lett. 75, 3969 (1995).

[4] A. L. Fetter, Rev. Mod. Phys. 81, 647 (2009).

[5] N. R. Cooper, Adv. Phys. 57, 593 (2008).

[6] E. J. Yarmchuk, M. J. V. Gordon, and R. E. Packard, Phys. Rev. Lett. 43, 214 (1979).

[7] K. W. Madison, F. Chevy, W. Wohlleben, and J. Dalibard, J. Mod. Opt. 47, 2715 (2000).

[8] K. W. Madison, F. Chevy, W. Wohlleben, and J. Dalibard, Phys. Rev. Lett. 84, 806 (2000).

[9] J. R. Abo-Shaeer, C. Raman, J. M. Vogels, and W. Ketterle, Science 292, 476 (2001).

[10] N. R. Cooper, E. H. Rezayi, and S. H. Simon, Phys. Rev. Lett. 95, 200402 (2005).

[11] J. Zhang and H. Zhai, Phys. Rev. Lett. 95, 200403 (2005).

[12] J. Radić, T. A. Sedrakyan, I. B. Spielman, and V. Galitski, Phys. Rev. A 84, 063604 (2011).

[13] E. A. L. Henn, J. A. Seman, G. Roati, K. M. F. Magalhães, and V. S. Bagnato, Phys. Rev. Lett. 103, 045301 (2009).

[14] R. P. Teles, F. E. A. dos Santos, M. A. Caracanhas, and V. S. Bagnato, Phys. Rev. A 87, 033622 (2013).

[15] S. S. Botelho and C. A. R. Sá de Melo, Phys. Rev. Lett. 96, 040404 (2006).

[16] M. Lu, N. Q. Burdick, S. H. Youn, and B. L. Lev, Phys. Rev. Lett. 107, 190401 (2011).

[17] K. Aikawa, A. Frisch, M. Mark, S. Baier, A. Rietzler, R. Grimm, and F. Ferlaino, Phys. Rev. Lett. 108, 210401 (2012).
[18] K. Kasamatsu, M. Tsubota, and M. Ueda, Phys. Rev. Lett. 93, 250406 (2004).

[19] P. Kuopanportti, J. A. M. Huhtamäki, and M. Möttönen, Phys. Rev. A 85, 043613 (2012).

[20] M. Eto and M. Nitta, Phys. Rev. A 85, 053645 (2012).

[21] M. Cipriani and M. Nitta, Phys. Rev. Lett. 111, 170401 (2013).

[22] C.-F. Liu, H. Fan, S.-C. Gou, and W.-M. Liu, Sci. Rep. 4, 4224 (2014).

[23] M. Eto, K. Kasamatsu, M. Nitta, H. Takeuchi, and M. Tsubota, Phys. Rev. A 83, 063603 (2011).

[24] A. Aftalion, P. Mason, and J. Wei, Phys. Rev. A 85, 033614 (2012).

[25] A. Chaves, F. M. Peeters, G. A. Farias, and M. V. Milošević, Phys. Rev. B 83, 054516 (2011).

[26] A. Chaves, L. Komendová, M. V. Milošević, J. S. Andrade, G. A. Farias, and F. M. Peeters, Phys. Rev. B 83, 214523 (2011).

[27] A. R. P. Lima and A. Pelster, Phys. Rev. A 86, 063609 (2012).

[28] A. L. Gaunt, T. F. Schmidutz, I. Gotlibovych, R. P. Smith, and Z. Hadzibabic, Phys. Rev. Lett. 110, 200406 (2013).

[29] L. Chomaz, L. Corman, T. Bienaimé, R. Desbuquois, C. Weitenberg, S. Nascimbène, J. Beugnon, and J. Dalibard, Nat. Commun. 6, 6162 (2015).

[30] D. A. Butts and D. S. Rokhsar, Nature (London) 397, 327 (1999).

[31] M. Linn and A. L. Fetter, Phys. Rev. A 60, 4910 (1999).

[32] R. P. Feynman, in Progress in Low Temperature Physics, Vol. 1 (North Holland, Amsterdam, 1955), pp. 17-53.

[33] M. R. Matthews, B. P. Anderson, P. C. Haljan, D. S. Hall, M. J. Holland, J. E. Williams, C. E. Wieman, and E. A. Cornell, Phys. Rev. Lett. 83, 3358 (1999).

[34] V. A. Schweigert, F. M. Peeters, and P. S. Deo, Phys. Rev. Lett. 81, 2783 (1998). 
[35] B. Xu, M. V. Milošević, S.-H. Lin, F. M. Peeters, and B. Jankó, Phys. Rev. Lett. 107, 057002 (2011).

[36] T. Cren, L. Serrier-Garcia, F. Debontridder, and D. Roditchev, Phys. Rev. Lett. 107, 097202 (2011).

[37] A. Aftalion and I. Danaila, Phys. Rev. A 69, 033608 (2004).

[38] I. Danaila, Phys. Rev. A 72, 013605 (2005).

[39] A. L. Fetter, B. Jackson, and S. Stringari, Phys. Rev. A 71, 013605 (2005).

[40] K. Kasamatsu and M. Tsubota, Phys. Rev. Lett. 97, 240404 (2006).

[41] R. Geurts, M. V. Milošević, and F. M. Peeters, Phys. Rev. A 78, 053610 (2008).
[42] G. Thalhammer, G. Barontini, L. De Sarlo, J. Catani, F. Minardi, and M. Inguscio, Phys. Rev. Lett. 100, 210402 (2008).

[43] S. B. Papp, J. M. Pino, and C. E. Wieman, Phys. Rev. Lett. 101, 040402 (2008).

[44] J. Garaud, J. Carlström, and E. Babaev, Phys. Rev. Lett. 107, 197001 (2011).

[45] V. Stanev and Z. Tešanović, Phys. Rev. B 81, 134522 (2010).

[46] S.-Z. Lin and X. Hu, Phys. Rev. Lett. 108, 177005 (2012).

[47] N. V. Orlova, A. A. Shanenko, M. V. Milošević, F. M. Peeters, A. V. Vagov, and V. M. Axt, Phys. Rev. B 87, 134510 (2013).

[48] U. R. Fischer, Phys. Rev. A 73, 031602(R) (2006).

[49] R. K. Kumar and P. Muruganandam, Eur. Phys. J. D 68, 289 (2014). 